\title{
Perturbation Analysis with Approximate Integration for Propagation Mode in Two-Dimensional Two-Slab Waveguides
}

\author{
Naofumi Kitsunezaki \\ Department of Integrated Information Technology, College of Science and Engineering, Aoyama Gauin University, \\ Sagamihara-shi, Kanagawa 252-5258, Japan \\ Correspondence should be addressed to Naofumi Kitsunezaki; kitsunezaki@it.aoyama.ac.jp
}

Received 23 May 2013; Accepted 14 July 2013

Academic Editors: Z. Chen, S. Wade, and D.-n. Wang

Copyright (c) 2013 Naofumi Kitsunezaki. This is an open access article distributed under the Creative Commons Attribution License, which permits unrestricted use, distribution, and reproduction in any medium, provided the original work is properly cited.

\begin{abstract}
On the basis of perturbation expansion from a gapless system, we calculate the propagation constant and propagation mode wave function in two-dimensional two-slab waveguides with a core gap small enough that there is only one propagation mode. We also perform calculations without the approximation for comparison. Our result shows that first-order perturbation contains the firstorder Taylor expansion of (core gap)/(core width), and when the integration of the perturbation is suitably approximated, the result of the first-order perturbation is the same as that of the first-order Taylor expansion of (core gap)/(core width).
\end{abstract}

\section{Introduction}

When monochromatic light enters into one of the cores of a waveguide array in which each core has the same width and the same gap, the light propagates to adjacent cores in turn, and its trajectory becomes V-shaped. Such optical behavior has been theoretically and numerically analyzed. Theoretical analysis includes matrix method [1], the coupled mode equation, and the coupled power equation [2-9]. In the coupling mode equations, it is assumed that there is at least one propagation mode for each core and that all of the propagation modes are independent from each other.

This assumption is invalid in the case that the number of independent propagation modes is less than the number of cores. Such a situation can occur when the core gap becomes small enough that the index distribution is approximated by a gapless core. When such a situation occurs, analysis based on the coupled mode or the coupled power equation is invalid. Our interest is finding a simple method to analyze an optical behavior in such a situation.

An optical behavior for parallel slab waveguides with small core gap was theoretically analyzed using even and odd mode analysis, which was called supermode analysis later [3-5]. In this analysis, the exact solution for the Maxwell's equations with the index distribution for two parallel slab waveguides, which becomes essentially one for Schrödinger equation for double finite wells in the field of quantum mechanics, is used to analyze the optical behavior. However, the method using supermodes is not useful when the number of cores increases, because the solution must be individually expressed in every core and clad.

In the field of quantum mechanics, perturbation theory is widely used as an approximation [10, 11]. In the field of electromagnetics and optics, it is also explained in [12], and applied to explain bend losses for a fiber [13, 14]. Coupled mode equation is also based on the perturbation theory [47]. The situation that we are interested in is suitable for using the perturbation theory.

In this paper, we calculate the supermode for a twodimensional two-slab waveguide that has a small core gap so that the optical system has only one propagation mode, using first-order perturbation from gapless system. We also calculate the same without approximation for comparison. In this way, we show that first-order perturbation with suitable approximate integration and the first-order Taylor expansion of (core gap)/(core width) give the same result. Furthermore, we discuss higher-order relationships between perturbation with suitable approximate integration and Taylor expansion. 


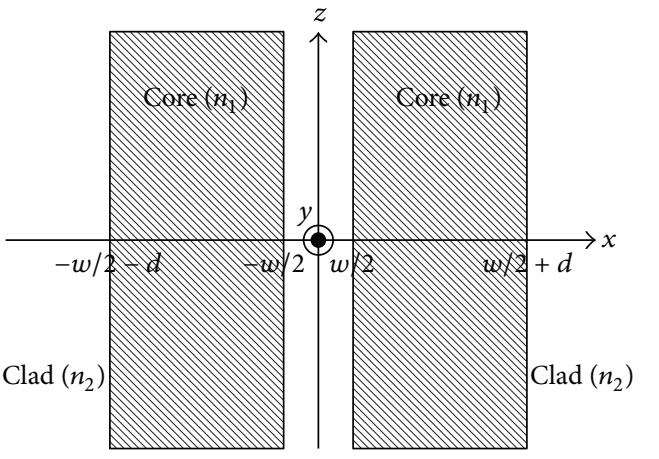

(a)

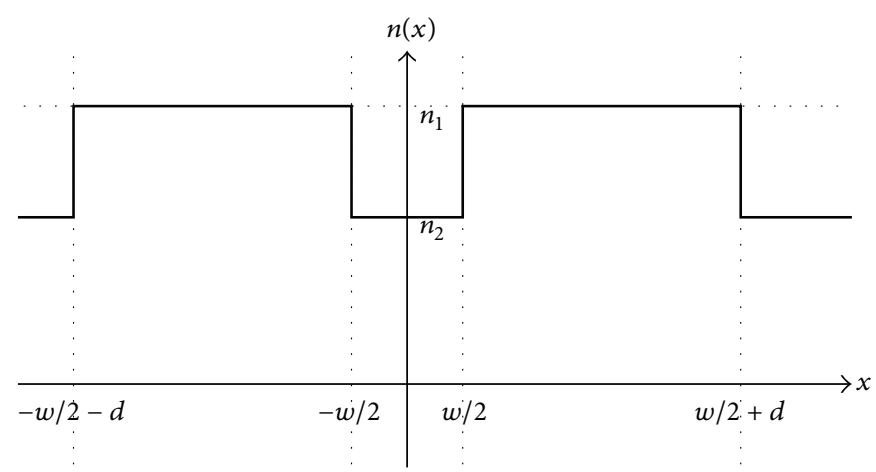

(b)

FIGURE 1: Optical system configuration (a) and its index distribution on $z=$ const. line (b).

\section{Model}

For our optical model of two cores with a gap, the spatial configuration of the cores and clads are shown in the left panel of Figure 1. There exist two cores with the same index $n_{1}$ and the same core width $d$, and clads with index $n_{2}$, where $n_{1}$ and $n_{2}$ are constants, and $n_{1}>n_{2}$. The core width $d$, core gap $w$, and indices $n_{1}$ and $n_{2}$ are chosen so that there is only one propagation mode. The core is configured in $w / 2<x<$ $w / 2+d$ and $-w / 2-d<x<-w / 2$. In other words, the index distribution $n(x)$ is set as

$$
n(x)= \begin{cases}n_{2}, & |x|<\frac{w}{2}, \\ n_{1}, & \frac{w}{2}<|x|<\frac{w}{2}+d, \\ n_{2}, & \frac{w}{2}+d<|x|,\end{cases}
$$

which is constant except for $x= \pm w / 2$ and $x= \pm(w / 2+d)$ and is illustrated in the right panel of Figure 1. The index distribution is constant in the $y$ and $z$ directions. The $z$ direction is defined as the direction of light propagation, and $\omega$ is used to denote the angular frequency of the monochromatic light.

Since the index distribution has no variation in the $y$ direction, electromagnetic fields do not depend on $y$. For the electric field $\mathbf{E}(t, x, z)$ and the magnetic field $\mathbf{H}(t, x, z)$, the time variable $t$ can be separated from the spatial variables as $\mathbf{E}(t, x, z)=\exp (i \omega t) \mathbf{e}(x, z)$ and $\mathbf{H}(t, x, z)=\exp (i \omega t) \mathbf{h}(x, z)$, because we are interested in monochromatic light with angular frequency $\omega$. Then, from Faraday's and AmpéreMaxwell's laws, components of the electric and magnetic fields have the following relationships:

$$
\begin{gathered}
h_{x}(x, z)=-\frac{i}{\omega \mu_{0}} \partial_{z} e_{y}(x, z), \\
h_{y}(x, z)=\frac{i}{\omega \mu_{0}}\left[\partial_{z} e_{x}(x, z)-\partial_{x} e_{z}(x, z)\right], \\
h_{z}(x, z)=\frac{i}{\omega \mu_{0}} \partial_{x} e_{y},
\end{gathered}
$$

$$
\begin{gathered}
e_{x}(x, z)=\frac{i}{\omega \varepsilon_{0} n(x)^{2}} \partial_{z} h_{y}(x, z), \\
e_{y}(x, z)=-\frac{i}{\omega \varepsilon_{0} n(x)^{2}}\left[\partial_{z} h_{x}(x, z)-\partial_{x} h_{z}(x, z)\right], \\
e_{z}(x, z)=-\frac{i}{\omega \varepsilon_{0} n(x)^{2}} \partial_{x} h_{y}(x, z) .
\end{gathered}
$$

It is straightforward to see that (2) to (7) are also solutions to the chargeless Gauss's law for electric and magnetic fields, which are expressed by the rest of Maxwell's equations. Equations (3) and (6) can be written as the second-order differential equations

$$
\begin{aligned}
& {\left[\partial_{x}^{2}+\partial_{z}^{2}+\frac{n(x)^{2}}{\lambda^{2}}\right] h_{y}(x, z)=0,} \\
& {\left[\partial_{x}^{2}+\partial_{z}^{2}+\frac{n(x)^{2}}{\lambda^{2}}\right] e_{y}(x, z)=0,}
\end{aligned}
$$

by using (5) and (7) and (2) and (4), respectively, where the derivative of $n(x)$ is neglected in (9) because $n(x)$ is constant except at some discrete points. Since (8) and (9) are basically the same differential equation, we introduce the field $\Psi(x, z)$ instead of $h_{y}(x, z)$ and $e_{y}(x, z)$. Equations (8) and (9) are usually solved by separation of variables:

$$
\Psi(x, z)=\psi(x) \phi(z) .
$$

So

$$
\begin{gathered}
\partial_{z}^{2} \phi(z)=\frac{e}{\hbar^{2}} \phi(z), \\
{\left[-\partial_{x}^{2}-\frac{n(x)^{2}}{\hbar^{2}}\right] \psi(x)=\frac{e}{\hbar^{2}} \psi(x),}
\end{gathered}
$$

where $e$ is a constant. In (11), $e$ obviously cannot be positive if the region of $z$ is $-\infty<z<\infty$, because positive $e$ makes $\phi(z)$ diverge in $z \rightarrow \infty$ or $z \rightarrow-\infty$. For any negative $e$, there exists a positive $\beta$ such that

$$
e=-\beta^{2}
$$


where $\beta$ is the propagation constant, and the solution of (11) represents propagation in the positive or negative $z$ direction with $\beta=[-e]^{1 / 2}$.

Equation (12) is essentially the one-dimensional Schrödinger equation $[10,11]$, which is an eigenfunction problem, where $e$ is the eigenvalue and $\psi$ is the eigenfunction of the operator:

$$
-\partial_{x}^{2}-\frac{n(x)^{2}}{\lambda^{2}}
$$

Equation (12) has nontrivial solutions for special values $e$ in $-n_{1}^{2}<e<-n_{2}^{2}$ (propagation mode solution), and two nontrivial solutions exist for each $e$ in $-n_{2}^{2}<e$ (radiation mode solution).

In the following section, we solve (12) by using first-order perturbation with a suitable approximation integration in order to calculate the difference of its propagation constant and propagation mode solution from that of a gapless system. In Section 4, we solve (12) without approximation in order to obtain the first-order Taylor expansion of $w / d$, and then we compared the result with those obtained in Section 3. The final section is devoted to summary and discussion.

\section{Solution with First-Order Perturbation}

To solve (12), we decompose the square of the index distribution $-n(x)^{2}$ into the square of the index distribution for $w=0$ $\left(-n_{0}(x)^{2}\right)$ and the rest $\left(-n_{p}(x)^{2}\right)$ as

$$
-n(x)^{2}=-n_{0}(x)^{2}-n_{p}(x)^{2},
$$

where

$$
\begin{gathered}
n_{0}(x)= \begin{cases}n_{1}, & \left|x-\frac{w}{2}\right|<d, \\
n_{2}, & d<\left|x-\frac{w}{2}\right|,\end{cases} \\
n_{p}(x)= \begin{cases}{\left[n_{1}^{2}-n_{2}^{2}\right]^{1 / 2},} & -\frac{w}{2}-d<x<\frac{w}{2}-d, \\
-\left[n_{1}^{2}-n_{2}^{2}\right]^{1 / 2}, & -\frac{w}{2}<x<\frac{w}{2}, \\
0, & \text { otherwise. }\end{cases}
\end{gathered}
$$

Figure 2 shows the distribution of $n_{0}(x)$ (solid line) and $n_{p}(x)$ (dotted line) on the $z=$ const. line. The index distribution of $n_{0}$ represents the core that is laid on $w / 2-d<x<w / 2+$ $d$. Instead of directly solving (12), we use the complete set of solutions for

$$
\left[-\partial_{x}^{2}-\frac{n_{0}(x)^{2}}{\lambda^{2}}\right] v(x)=\frac{e}{\lambda^{2}} v(x),
$$

in order to solve (12) perturbatively. All independent solutions for (17) are

$$
V_{0}(x)=\left\{\begin{array}{cc}
v_{0} \cos \left[\frac{P_{0}(2 x-w)}{d}\right], & \left|x-\frac{w}{2}\right|<d, \\
v_{0} \cos \left(2 P_{0}\right) & \\
\quad \times \exp \left[-\frac{Q_{0}(|2 x-w|-2 d)}{d}\right], & d<\left|x-\frac{w}{2}\right|,
\end{array}\right.
$$

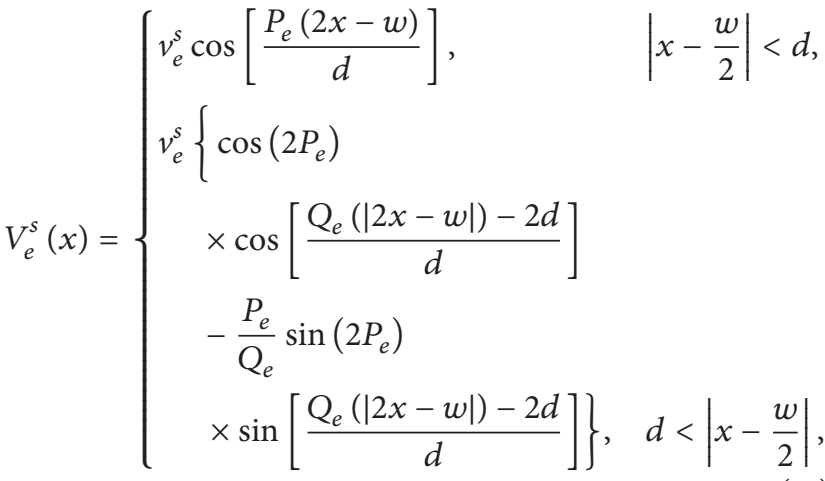

$$
V_{e}^{a}(x)= \begin{cases}v_{e}^{a} \sin \left[\frac{P_{e}(2 x-w)}{d}\right], & \left|x-\frac{w}{2}\right|<d, \\ v_{e}^{a} \operatorname{sign}(x) & \times\left\{\sin \left(2 P_{e}\right)\right. \\ & \times \cos \left[\frac{Q_{e}(|2 x-w|-2 d)}{d}\right] \\ & +\frac{P_{e}}{Q_{e}} \cos \left(2 P_{e}\right) \\ & \left.\times \sin \left[\frac{Q_{e}(|2 x-w|-2 d)}{d}\right]\right\},\end{cases}
$$

where

$$
\begin{gathered}
P_{0}=\frac{\left[n_{1}^{2}+e_{0}\right]^{1 / 2} d}{2 \lambda}, \quad Q_{0}=\frac{\left[-\left(n_{2}^{2}+e_{0}\right)\right]^{1 / 2} d}{2 \lambda}, \\
P_{e}=\frac{\left[n_{1}^{2}+e\right]^{1 / 2} d}{2 \lambda}, \quad Q_{e}=\frac{\left[n_{2}^{2}+e\right]^{1 / 2} d}{2 \lambda},
\end{gathered}
$$

and $e_{0}$ (or the pair of $P_{0}$ and $Q_{0}$ ) in (21) satisfy

$$
\begin{gathered}
Q_{0}=P_{0} \tan \left(2 P_{0}\right), \\
P_{0}^{2}+Q_{0}^{2}=V^{2},
\end{gathered}
$$

where

$$
V=\frac{\left[n_{1}^{2}-n_{2}^{2}\right]^{1 / 2} d}{2 \lambda} .
$$




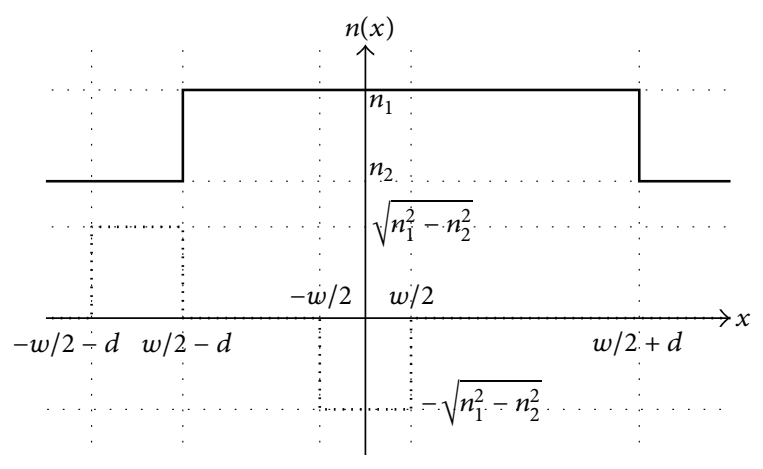

FIGURE 2: Decomposed index distributions. Solid line represents $n_{0}(x)$, and dotted line represents $n_{p}(x)$.

In (22), $e$ is in the region

$$
-n_{2}^{2}<e<0
$$

$V_{0}$ represents the propagation mode, and $V_{e}^{s}$ and $V_{e}^{a}$ represent symmetric and antisymmetric radiation modes according to $e$, respectively. For the reasons stated in the introduction, we are interested in the case that the propagation mode of the optical system has only one independent mode. Therefore, we examine the case where the normalized propagation mode solution of (18) is $V_{0}$ only.

Here, let us define the inner product of fields $f \cdot g$ as

$$
f \cdot g=\frac{1}{d} \int_{-\infty}^{\infty} d x f(x) g(x)
$$

Then, $v_{0}, v_{e}^{s}$, and $v_{e}^{a}$ are defined so that $V_{0}, V_{e}^{s}$, and $V_{e}^{a}$ are normalized to

$$
\begin{gathered}
V_{0} \cdot V_{0}=1, \\
V_{e}^{p} \cdot V_{f}^{q}=\delta^{p q} \delta(e-f),
\end{gathered}
$$

where $p, q \in\{s, a\}$ (note that $V_{0}, V_{e}^{s}$ and $V_{e}^{a}$ can be regarded as eigenfunctions of the eigenequation (17), and their eigenvalues are $e_{0}, e$, and $e$, respectively. In addition to the relationships in (28), $V_{0} \cdot V_{e}^{s}=0$ and $\left.V_{0} \cdot V_{e}^{a}=0[10,11]\right)$. For instance, $v_{0}$ is

$$
v_{0}=\left[\frac{2 Q_{0}}{2 Q_{0}+1}\right]^{1 / 2}
$$

In perturbation theory, the propagation mode solution for (12) is determined by using (18) to (20), which results in

$$
\begin{gathered}
\left\{\left[-\partial_{x}^{2}-\frac{n_{0}(x)^{2}}{\lambda^{2}}\right]-\frac{n_{p}(x)^{2}}{\lambda^{2}}\right\}\left[V_{0}(x)+\Delta V(x)\right] \\
=\frac{e_{0}+\Delta e}{\lambda^{2}}\left[V_{0}(x)+\Delta V(x)\right] .
\end{gathered}
$$

The results of the first-order perturbation for $\Delta e$ and $\Delta V$ are as follows $[10,11]$ :

$$
\begin{gathered}
\Delta e=-V_{0} \cdot\left(n_{p}^{2} V_{0}\right), \\
V_{0} \cdot \Delta V=0, \\
V_{e}^{p} \cdot \Delta V=\frac{V_{e}^{p} \cdot\left(n_{p}^{2} V_{0}\right)}{e-e_{0}},
\end{gathered}
$$

where

$$
\left(n_{p}^{2} V_{0}\right)(x)=n_{p}(x)^{2} V_{0}(x)
$$

In (33), $V_{e}^{s} \cdot \Delta V$ and $V_{e}^{a} \cdot \Delta V$ mean the $V_{e}^{s}$ and $V_{e}^{a}$ components of $\Delta V$, respectively. Note that for any field $f(x)$, the inner product with $n_{p}^{2} V_{0}$ is

$$
\begin{aligned}
f \cdot\left(n_{p}^{2} V_{0}\right)= & \frac{n_{1}^{2}-n_{2}^{2}}{d}\left[\int_{-(w / 2)-d}^{(w / 2)-d} d x f(x) V_{0}(x)\right. \\
& \left.-\int_{-(w / 2)}^{w / 2} d x f(x) V_{0}(x)\right], \\
= & \left(n_{1}^{2}-n_{2}^{2}\right)\left[f\left(\frac{w}{2}-d\right) V_{0}\left(\frac{w}{2}-d\right)\right. \\
& +o\left(\frac{w}{d}\right)^{2},
\end{aligned}
$$

which means that it contains the first-order Taylor expansion of $w / d$. In the deformation from (35) to (36), we use the Euler method for the integration. Using (18) through (20), we obtain the first-order Taylor expansion of $w / d$ for $\Delta e, V_{e}^{s} \cdot \Delta V$, and $V_{e}^{a} \cdot \Delta V$ from (31) and (33), respectively, as follows:

$$
\begin{gathered}
\Delta e=v_{0}^{2}\left(n_{1}^{2}-n_{2}^{2}\right)\left[1-\cos ^{2}\left(2 P_{0}\right)\right] \\
=\frac{2 Q_{0}\left(n_{1}^{2}-n_{2}^{2}\right) \sin ^{2}\left(2 P_{0}\right)}{2 Q_{0}+1} \frac{w}{d}, \\
V_{e}^{s} \cdot \Delta V=-v_{0} v_{s}^{e} \frac{\left(n_{1}^{2}-n_{2}^{2}\right)\left[1-\cos \left(2 P_{0}\right) \cos \left(2 P_{e}\right)\right]}{e-e_{0}} \frac{w}{d},
\end{gathered}
$$

$$
V_{e}^{a} \cdot \Delta V=-v_{0} v_{s}^{e} \frac{\left(n_{1}^{2}-n_{2}^{2}\right) \sin \left(2 P_{0}\right) \cos \left(2 P_{e}\right)}{e-e_{0}} \frac{w}{d} .
$$




\section{Solution without Approximation}

When the exact eigenvalue of the propagation mode of (12) is $e_{w}$, its eigenfunction is

$$
W(x)=\left\{\begin{array}{cl}
w_{0} \cosh \left(\frac{2 Q_{w} x}{d}\right), & |x|<\frac{w}{2}, \\
w_{0}\left\{\cosh \left(\frac{Q_{w} w}{d}\right)\right. & \\
\times \cos \left[\frac{P_{w}(2|x|-w)}{d}\right] & \\
+\frac{Q_{w}}{P_{w}} \sinh \left(\frac{Q_{w} w}{d}\right) & \\
\left.\times \sin \left[\frac{P_{w}(2|x|-w)}{d}\right]\right\}, & \frac{w}{2}<|x|<\frac{w}{2}+d, \\
w_{0}\left[\cosh \left(\frac{Q_{w} w}{d}\right) \cos \left(2 P_{w}\right)\right. & \\
+\frac{Q_{w}}{P_{w}} \sinh \left(\frac{Q_{w} w}{d}\right) & \\
\left.\times \sin \left(2 P_{w}\right)\right] & \\
\times \exp \left[-\frac{Q_{w}(2|x|-2 d-w)}{d}\right], & \frac{w}{2}+d<|x|,
\end{array}\right.
$$

where

$$
P_{w}=\frac{\left[n_{1}^{2}+e_{w}\right]^{1 / 2} d}{2 \lambda}, \quad Q_{w}=\frac{\left[-\left(n_{2}^{2}+e_{w}\right)\right]^{1 / 2} d}{2 \lambda} .
$$

$P_{w}$ and $Q_{w}$ satisfy the relationships

$$
P_{w}\left[P_{w} \sin \left(2 P_{w}\right)-Q_{w} \cos \left(2 P_{w}\right)\right]
$$

$$
\begin{aligned}
& V_{e}^{s} \cdot W_{0} \\
& =v_{0} v_{e}^{s}\left(\frac{Q_{w}\left[1+\cosh \left(2 P_{e} w / d\right)\right] \sin \left(Q_{w} w / d\right)+P_{e} \sinh \left(2 P_{e} w / d\right) \cos \left(Q_{w} w / d\right)}{2\left(P_{e}^{2}+Q_{w}^{2}\right)}\right. \\
& +\frac{P_{w} \cosh \left(Q_{w} w / d\right) \cos \left(2 P_{w}\right)+Q_{w} \sinh \left(Q_{w} w / d\right) \sin \left(2 P_{w}\right)}{P_{w}\left(Q_{e}^{2}+Q_{w}^{2}\right)} \\
& \times\left\{\frac{\left[1+2 \cos \left(2 Q_{e} w / d\right)-\exp \left(2 Q_{w} w / d\right)\right]\left[Q_{w} \cos \left(2 P_{e}\right)-P_{e} \sin \left(2 P_{e}\right)\right]}{2}\right. \\
& \left.+\frac{\left[Q_{e}^{2} \cos \left(2 P_{e}\right)+P_{e} Q_{w} \sin \left(2 P_{e}\right)\right] \sin \left(2 Q_{e} w / d\right)}{Q_{e}}\right\} \\
& -\frac{1}{P_{e}^{2}-P_{w}^{2}}\left[P _ { w } \operatorname { c o s h } ( \frac { Q _ { w } w } { d } ) \left(\frac{\left[1+\cos \left(2 P_{w} w / d\right)\right] \sin \left(2 P_{w}\right) \cos \left(2 P_{e}\right)}{2}\right.\right. \\
& -\frac{\sin \left(2 P_{w} w / d\right) \cos \left(2 P_{w}\right) \cos \left(2 P_{e}\right)}{2}
\end{aligned}
$$
with gap $w$. we obtain (23), we obtain (37) from (45). Because of (13), the first-order perturbation and the first-order Taylor expansion of $w / d$ give the same propagation constant for a two-core optical system

$V_{e}^{s} \cdot W$ and $V_{e}^{a} \cdot W$ are directly derived, which results in

$$
P_{w}^{2}+Q_{w}^{2}=V^{2} \text {, }
$$

such that $W(x)$ and its derivative are continuous for any $x$. Note that $W_{0}(x)$ agrees with $V_{0}(x)$ in the limit of $w / d=0$, and $w_{0}$ becomes $v_{0}$ when $w / d=0$. When $w / d \neq 0$, the ationships between $W(x)$ and $\Delta V(x)$ and between $e_{w}$ and Substituting (44) into (42) by using (41), and applying (23),

$$
\begin{gathered}
\frac{\left[2 P_{0} \sin \left(2 P_{0}\right)+\cos \left(2 P_{0}\right)\right] V^{2} \Delta e}{2\left(n_{1}^{2}-n_{2}^{2}\right) \sin \left(2 P_{0}\right) \cos \left(2 P_{0}\right)}[1+O(\Delta e)] \\
\quad=V^{2} Q_{0} \sin \left(2 P_{0}\right) \frac{w}{d}\left[1+O\left(\frac{w}{d}, \Delta e\right)\right] .
\end{gathered}
$$




$$
\begin{aligned}
& -\frac{P_{e}}{P_{w}}\left\{\frac{\left[1+\cos \left(2 P_{w} w / d\right)\right] \cos \left(2 P_{w}\right) \cos \left(2 P_{e}\right)}{2}\right. \\
& \left.\left.+\frac{\sin \left(2 P_{w} w / d\right) \sin \left(2 P_{w}\right) \sin \left(2 P_{e}\right)}{2}-\sin \left(\frac{2 P_{e} w}{d}\right)\right\}\right) \\
& +Q_{w} \sinh \left(\frac{Q_{w} w}{d}\right)\left\{\frac{\left[1+\cos \left(2 P_{e} w / d\right)\right]\left[1-\cos \left(2 P_{w}\right) \cos \left(2 P_{e}\right)\right]}{2}\right. \\
& -\frac{\sin \left(2 P_{w} w / d\right) \sin \left(2 P_{w}\right) \sin \left(2 P_{e}\right)}{2}+\cos \left(\frac{2 P_{e} w}{d}\right) \\
& -\cos \left(\frac{2 P_{w} w}{d}\right)-\frac{\left[1+\cos \left(2 P_{w} w / d\right)\right] \sin \left(2 P_{w}\right) \sin \left(2 P_{e}\right)}{2} \\
& \left.\left.+\frac{\sin \left(2 P_{w} w / d\right) \cos \left(2 P_{w}\right) \cos \left(2 P_{e}\right)}{2}\right\}\right] \\
& -\left(\left(\left\{\left[P_{w} \sin \left(\frac{2 P_{w} w}{d}\right)-Q_{e} \sin \left(\frac{2 Q_{e} w}{d}\right)\right] \cosh \left(\frac{Q_{w} w}{d}\right)\right.\right.\right. \\
& \left.\left.-Q_{w}\left[\cos \left(\frac{2 Q_{e} w}{d}\right)-\cos \left(\frac{2 P_{w} w}{d}\right)\right] \sinh \left(\frac{Q_{w} w}{d}\right)\right\} \cos \left(2 P_{w}\right) \cos \left(2 P_{e}\right)\right) \\
& \left.\times\left(2\left(Q_{e}^{2}-P_{w}^{2}\right)\right)^{-1}\right) \\
& -\left(\left(\left\{P_{e}\left[\sin \left(\frac{2 P_{w} w}{d}\right)+\frac{P_{w}}{Q_{e}} \sin \left(\frac{2 Q_{e} w}{d}\right)\right] \cosh \left(\frac{Q_{w} w}{d}\right)\right.\right.\right. \\
& \left.\left.-\frac{Q_{w} P_{e}}{P_{w}}\left[\cos \left(\frac{2 Q_{e} w}{d}\right)-\cos \left(\frac{2 P_{w} w}{d}\right)\right] \sinh \left(\frac{Q_{w} w}{d}\right)\right\} \sin \left(2 P_{w}\right) \sin \left(2 P_{e}\right)\right) \\
& \left.\times\left(2\left(Q_{e}^{2}-P_{w}^{2}\right)\right)^{-1}\right) \\
& -\left(\left(\left\{Q_{w}\left[\sin \left(\frac{2 P_{w} w}{d}\right)+\frac{Q_{e}}{P_{w}} \sin \left(\frac{2 Q_{e} w}{d}\right)\right] \sinh \left(\frac{Q_{w} w}{d}\right)\right.\right.\right. \\
& \left.\left.+\left[\cos \left(\frac{2 Q_{e} w}{d}\right)-\cos \left(\frac{2 P_{w} w}{d}\right)\right] \cosh \left(\frac{Q_{w} w}{d}\right)\right\} \sin \left(2 P_{w}\right) \cos \left(2 P_{e}\right)\right) \\
& \left.\times\left(2\left(Q_{e}^{2}-P_{w}^{2}\right)\right)^{-1}\right) \\
& +\left(\left(P _ { e } P _ { w } \left\{\frac{1}{P_{w}}\left[\sin \left(\frac{2 P_{w} w}{d}\right)+\frac{1}{Q_{e}} \sin \left(\frac{2 Q_{e} w}{d}\right)\right] \sinh \left(\frac{Q_{w} w}{d}\right)\right.\right.\right. \\
& \left.\left.+P_{e}\left[\cos \left(\frac{2 Q_{e} w}{d}\right)-\cos \left(\frac{2 P_{w} w}{d}\right)\right] \cosh \left(\frac{Q_{w} w}{d}\right)\right\} \cos \left(2 P_{w}\right) \sin \left(2 P_{e}\right)\right) \\
& \left.\left.\times\left(2\left(Q_{e}^{2}-P_{w}^{2}\right)\right)^{-1}\right)\right) \\
& =v_{e}^{a} v_{0}\left(-\frac{P_{e}\left[1-\cos \left(2 P_{e} w / d\right)\right] \cosh \left(Q_{w} w / d\right)+Q_{w} \sinh \left(Q_{w} w / d\right) \sin \left(2 P_{e} w / d\right)}{2\left(P_{e}^{2}+Q_{w}^{2}\right)}\right. \\
& +\frac{P_{w} \cosh \left(Q_{w} w / d\right) \cos \left(2 P_{w}\right)+Q_{w} \sinh \left(Q_{w} w / d\right) \sin \left(2 P_{w}\right)}{P_{w}\left(Q_{e}^{2}+Q_{w}^{2}\right)} \\
& \times\left\{\frac{\left[1-\cos \left(2 Q_{e} w / d\right)\right]\left[Q_{w} \sin \left(2 P_{e}\right)+P_{e} \cos \left(2 P_{e}\right)\right]}{2}\right. \\
& \left.+\frac{\left[Q_{e}^{2} \sin \left(2 P_{e}\right)-P_{e} Q_{w} \cos \left(2 P_{e}\right)\right] \sin \left(2 P_{e} w / d\right)}{4 Q_{e}}\right\}
\end{aligned}
$$




$$
\begin{aligned}
& +\frac{1-\cos \left(2 P_{e} w / d\right)}{2} \frac{P_{w} \cosh \left(Q_{w} w / d\right)\left\{P_{e}\left[1-\cos \left(2 P_{w}\right) \cos \left(2 P_{e}\right)\right]-P_{w} \sin \left(2 P_{w}\right) \sin \left(2 P_{e}\right)\right\}}{P_{w}\left(P_{e}^{2}-P_{w}^{2}\right)} \\
& +\frac{1-\cos \left(2 P_{e} w / d\right)}{2} \frac{Q_{w} \sinh \left(Q_{w} w / d\right)\left[P_{e} \cos \left(2 P_{w}\right) \sin \left(2 P_{e}\right)-P_{w} \sin \left(2 P_{w}\right) \cos \left(2 P_{e}\right)\right]}{P_{w}\left(P_{e}^{2}-P_{w}^{2}\right)} \\
& +\frac{\sin \left(2 P_{e} w / d\right)}{2} \frac{P_{w} \cosh \left(Q_{w} w / d\right)\left[P_{w} \sin \left(2 P_{w}\right) \cos \left(2 P_{e}\right)-P_{e} \cos \left(2 P_{w}\right) \sin \left(2 P_{e}\right)\right]}{P_{w}\left(P_{e}^{2}-P_{w}^{2}\right)} \\
& +\frac{\sin \left(2 P_{e} w / d\right)}{2} \frac{Q_{w} \sinh \left(Q_{w} w / d\right)\left\{P_{w}\left[1-\cos \left(2 P_{w}\right) \cos \left(2 P_{e}\right)\right]-P_{e} \sin \left(2 P_{w}\right) \sin \left(2 P_{e}\right)\right\}}{P_{w}\left(P_{e}^{2}-P_{w}^{2}\right)} \\
& -\left(\left(\operatorname { c o s h } ( \frac { Q _ { w } w } { d } ) \left\{P_{e}\left[\cos \left(2 P_{w}\right) \cos \left(2 P_{e}\right)-\cos \left(\frac{2 P_{w}(d-w)}{d}\right) \cos \left(\frac{2 P_{e}(d-w)}{d}\right)\right]\right.\right.\right. \\
& \left.\left.+P_{w}\left[\sin \left(2 P_{w}\right) \sin \left(2 P_{e}\right)-\sin \left(\frac{2 P_{w}(d-w)}{d}\right) \sin \left(\frac{2 P_{e}(d-w)}{d}\right)\right]\right\}\right) \\
& \left.\times\left(2\left(P_{e}^{2}-P_{w}^{2}\right)\right)^{-1}\right) \\
& +\left(\left(Q _ { w } \operatorname { s i n h } ( \frac { Q _ { w } w } { d } ) \left\{P_{e}\left[\cos \left(2 P_{w}\right) \sin \left(2 P_{e}\right)-\cos \left(\frac{2 P_{w}(d-w)}{d}\right) \sin \left(\frac{2 P_{e}(d-w)}{d}\right)\right]\right.\right.\right. \\
& \left.\left.-P_{w}\left[\sin \left(2 P_{w}\right) \cos \left(2 P_{e}\right)-\sin \left(\frac{2 P_{w}(d-w)}{d}\right) \cos \left(\frac{2 P_{0}(d-w)}{d}\right)\right]\right\}\right) \\
& \left.\times\left(2 P_{w}\left(P_{e}^{2}-P_{w}^{2}\right)\right)^{-1}\right) \\
& +\left(\left(\operatorname { s i n h } ( \frac { Q _ { w } w } { d } ) \left\{P_{e}\left[\sin \left(\frac{2 P_{w} w}{d}\right)+\frac{P_{w}}{Q_{e}} \sin \left(\frac{2 Q_{e} w}{d}\right)\right] \cos \left(2 P_{w}\right) \cos \left(2 P_{e}\right)\right.\right.\right. \\
& \left.\left.-\frac{Q_{w}}{P_{w}}\left[P_{w} \sin \left(\frac{2 P_{w} w}{d}\right)+Q_{e} \sin \left(\frac{2 Q_{e} w}{d}\right)\right] \sin \left(2 P_{w}\right) \sin \left(2 P_{e}\right)\right\}\right) \\
& \left.\times\left(2\left(Q_{e}^{2}-P_{w}^{2}\right)\right)^{-1}\right) \\
& +\left(\left(\operatorname { c o s h } ( \frac { Q _ { w } w } { d } ) \left\{Q_{e}\left[P_{w} \sin \left(\frac{2 P_{w} w}{d}\right)+Q_{e} \sin \left(\frac{2 Q_{e} w}{d}\right)\right] \cos \left(2 P_{w}\right) \sin \left(2 P_{e}\right)\right.\right.\right. \\
& \left.\left.\left.\left.-P_{e}\left[Q_{e} \sin \left(\frac{2 P_{w} w}{d}\right)+P_{w} \sin \left(\frac{2 Q_{e} w}{d}\right)\right] \sin \left(2 P_{w}\right) \cos \left(2 P_{e}\right)\right\}\right) \times\left(2 Q_{e}\left(Q_{e}^{2}-P_{w}^{2}\right)\right)^{-1}\right)\right) \text {. }
\end{aligned}
$$

From (46), we obtain the first-order Taylor expansion of $w / d$ of $V_{e}^{s} \cdot W$ and $V_{e}^{a} \cdot W$ after a straightforward but lengthy calculation:

$$
\begin{gathered}
V_{e}^{s} \cdot W=-v_{0} v_{e}^{s} \frac{\left(n_{1}^{2}-n_{2}^{2}\right)\left[1-\cos \left(2 P_{0}\right) \cos \left(2 P_{e}\right)\right]}{e-e_{0}} \frac{w}{d}, \\
V_{e}^{a} \cdot W=-v_{0} v_{e}^{a} \frac{\left(n_{1}^{2}-n_{2}^{2}\right) \sin \left(2 P_{0}\right) \cos \left(2 P_{e}\right)}{e-e_{0}} \frac{w}{d} .
\end{gathered}
$$

$V_{e}^{s} \cdot \Delta V$ and $V_{e}^{a} \cdot \Delta V$ are equal to $V_{e}^{s} \cdot W$ and $V_{e}^{a} \cdot W$, respectively, because of (32) and (43). As shown above, (38) and (39) completely coincide with (47) and (48), respectively.

\section{Conclusion and Discussion}

We have shown that in a two-dimensional two-slab waveguide system that has a small core gap so that the optical system has only one propagation mode, the supermode and its propagation constant calculated by first-order perturbation by the Euler method and the first-order Taylor expansion of $w / d$ for the exact solution give the same result up to $(w / d)^{1}$ order.

As for higher-order perturbation, the correction of $e_{w}$ by second-order perturbation is given by [10]

$$
\sum_{p \in\{s, a\}} \int_{-n_{2}^{2}}^{0} \operatorname{de\rho }(e) \frac{\left|V_{e}^{p} \cdot\left(n_{2}^{2} V_{0}\right)\right|^{2}}{e-e_{0}}
$$


where $\rho(e)$ represents mode density function, and the correction of $V_{e}^{p} \cdot W$ is

$$
\begin{aligned}
\sum_{q \in\{s, a\}} \int_{-n_{2}^{2}}^{0} d f \frac{V_{e}^{p} \cdot\left(n_{2}^{2} V_{f}^{q}\right) V_{f}^{q} \cdot\left(n_{2}^{2} V_{0}\right)}{\left(e-e_{0}\right)\left(f-e_{0}\right)} \\
-\frac{V_{e}^{p} \cdot\left(n_{2}^{2} V_{0}\right) V_{0} \cdot\left(n_{2}^{2} V_{0}\right)}{\left(e-e_{0}\right)^{2}} .
\end{aligned}
$$

The lowest order of $w / d$ for (49) and (50) is $O(w / d)^{2}$, because of the argument given in Section 3. Therefore, second-order perturbation with the Euler method is a part of the secondorder Taylor expansion of $w / d$. As shown in (36), the firstorder perturbation can include the second order of $w / d$. To obtain the second order of $w / d$ from the first-order perturbation, integration over $-w / 2<x<w / 2-d$ and $-w / 2<x<w / 2$ in the perturbation must be approximated by the trapezoidal rule instead of the Euler method. Thus, first-order perturbation by the trapezoidal rule in combination with a second-order perturbation by the Euler method gives the same result as the exact calculation given by the second-order Taylor expansion of $w / d$. In a similar way, the $n$ th-order coefficient of the Taylor expansion of $w / d$ is obtained from all orders of perturbation less than the $n$th order. In each order of perturbation, exact integration over $-w / 2-d<x<w / 2-d$ and $-w / 2<x<w / 2$ is not necessary; instead, a suitable approximation of integration including the $(w / d)^{n}$ order term is necessary. Thus, when an optical system has two cores with a small gap, it can be analyzed by perturbation using modes of an optical system with a gapless core with twice the width.

Obtaining the exact solution becomes time consuming as the number of cores increases. Even in such situations, perturbation analysis can be executed easily. Hence, perturbation analysis using suitable approximate integration is a promising candidate for approximation in the region $w / d \ll 1$ when the coupled mode or power mode equation is invalid because $w / d$ is very small.

\section{Acknowledgment}

The authors thank all members of the Tobe Laboratory in the Department of Integrated Information Technology, College of Science and Engineering, Aoyama Gakuin University, for comments and discussion.

\section{References}

[1] A. Ghatak and V. Lakshminarayanan, "Propagation characteristics of planar waveguides," in Optical Waveguides from Theory to Applied Technologies, M. L. Calvo and V. Lakshminarayanan, Eds., p. CRC press, New York, NY, USA, 2007.

[2] A. Yariv, "Coupling-mode theory for guided-wave optics," IEEE Journal of Quantum Electronics, vol. QE-9, no. 9, pp. 919-933, 1973.

[3] Y. Suematsu and K. Kishino, "Coupling coefficient in strongly coupled dielectric waveguides," Radio Science, vol. 12, no. 4, pp. 587-592, 1977.
[4] A. Hardy and W. Streifer, "Coupled mode theory of parallel waveguides," Journal of Lightwave Technology, vol. 3, no. 5, pp. 1135-1146, 1985.

[5] A. Hardy and W. Streifer, "Coupled modes of multiwaveguide system and phased arrays," Journal of Lightwave Technology, vol. 4, no. 1, pp. 90-99, 1986.

[6] H. A. Haus, W. P. Huang, S. Kawakami, and N. A. Whitaker, "Coupled-mode theory of optical waveguides," Journal of Lightwave Technology, vol. 5, no. 1, pp. 16-23, 1987.

[7] K. Yasumoto, "Coupled-mode formulation of parallel dielectric waveguides using singular perturbation technique," Microwave and Optical Technology Letters, vol. 4, no. 11, pp. 486-491, 1991.

[8] H. Yoshida, K. Hidaka, and N. Tsukada, "Proposal of optical waveguide array beam splitter and Mach-Zehnder optical interferometer," IEICE Technical Report OCS2010-36, 2010.

[9] A. Komiyama, "Energy conservation in a waveguide system with an imperfection core," The Papers of Technical Meeting on Electromagnetic Theory, IEEJ, EMT-11-131, 2011.

[10] L. I. Shiff, Quantum Mechanics, Mc-Graw Hill, Singapore, 1969.

[11] W. Greiner, Quantum Mechanics-An Introduction, Springer, Berlin, Germany, 1989.

[12] R. F. Harrington, Time-Harmonic Electromagnetic Fields, WileyInterscience, New York, NY, USA, 2001.

[13] C. Vassallo, "Perturbation of a LP mode of an optical fibre by a quasi-degenerate field: a simple formula," Optical and Quantum Electronics, vol. 17, no. 3, pp. 201-205, 1985.

[14] Q. Wang, G. Farrell, and T. Freir, “Theoretical and experimental investigations of macro-bend Losses for standard single mode fibers," Optics Express, vol. 13, no. 12, pp. 4476-4484, 2005. 

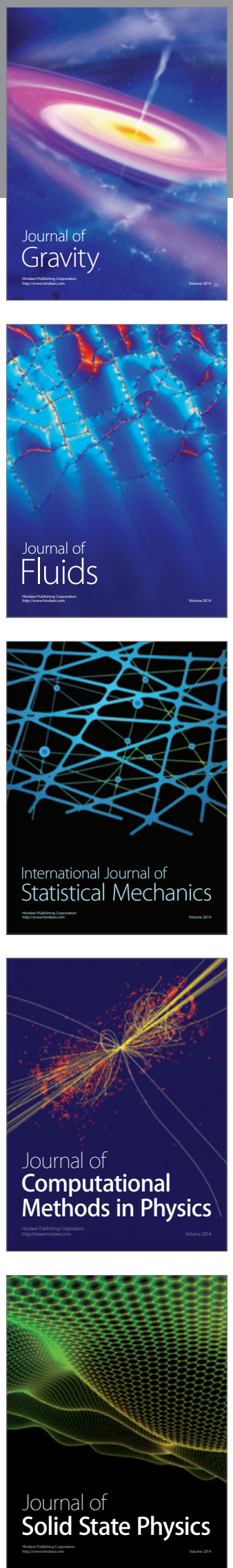

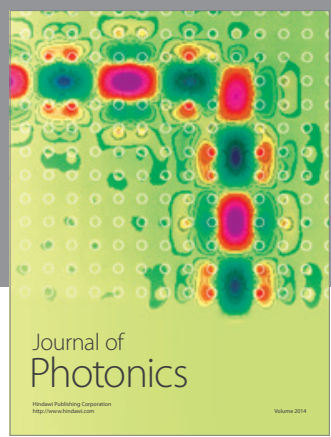

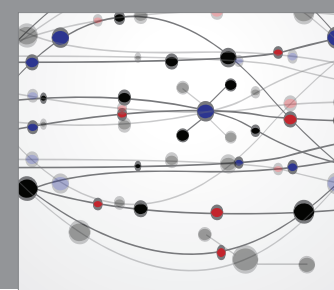

The Scientific World Journal

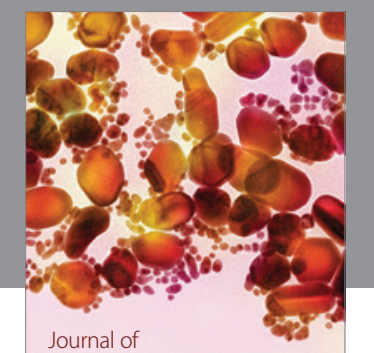

Soft Matter
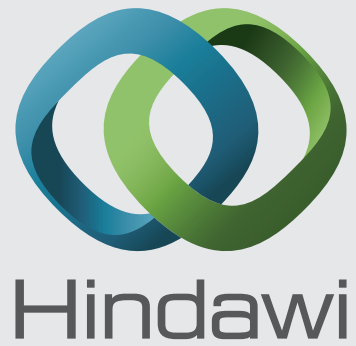

Submit your manuscripts at

http://www.hindawi.com
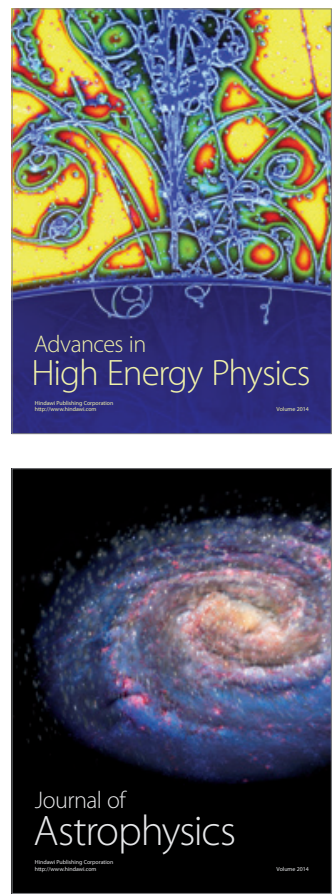
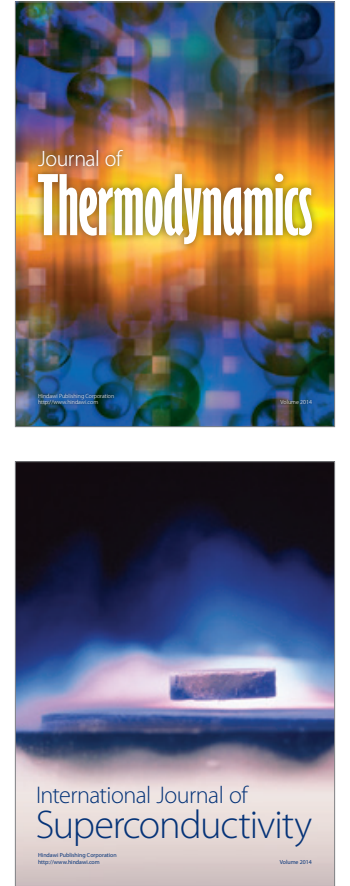
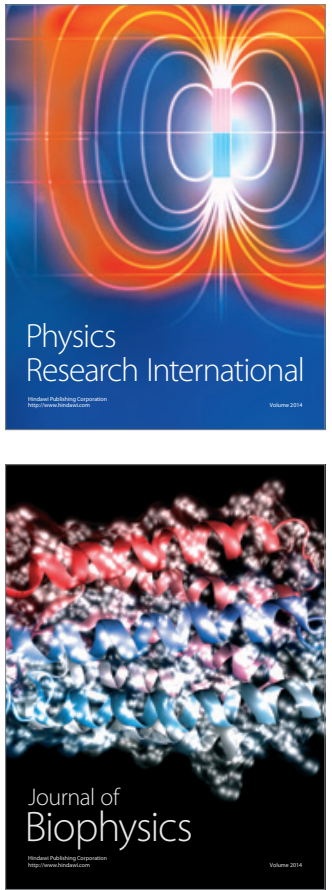
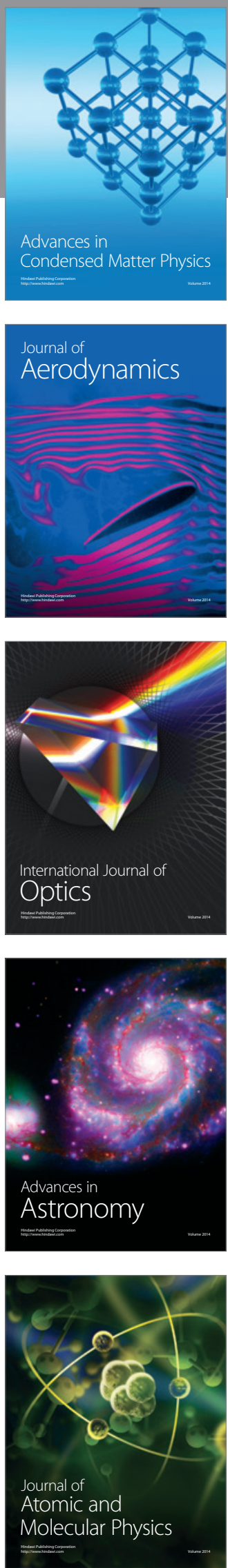